\title{
MaIn PRINCIPALS AND ISSUES OF Digital TWIN DeVElopMent For COMPLEX TeChNOlogical Processes
}

\author{
Kirill Ponomarev, Nikita Kudryashov, Nikita Popelnukha \& Vyacheslav Potekhin
}
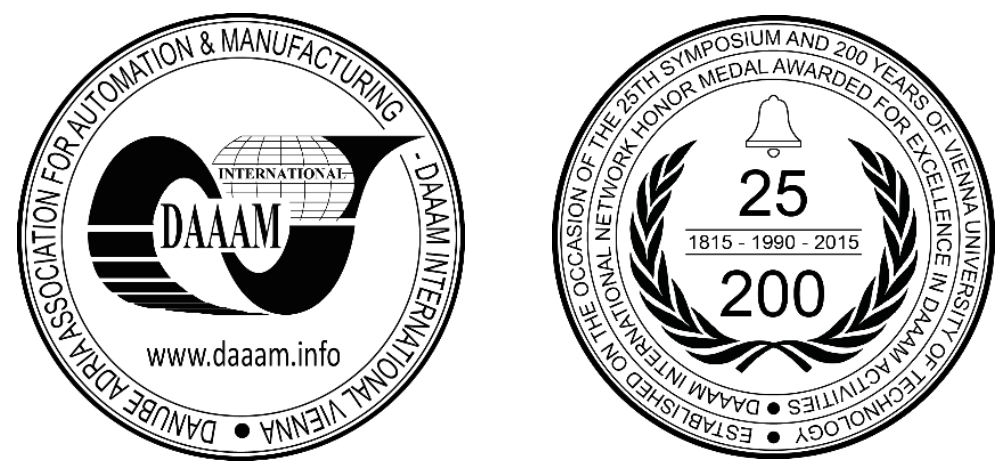

This Publication has to be referred as: Ponomarev, K[irill]; Kudryashov, N[ikita] \& Popelnukha, N[ikita] (2017). Main Principals and Issues of Digital Twin Development for Complex Technological Processes, Proceedings of the 28th DAAAM International Symposium, pp.0523-0528, B. Katalinic (Ed.), Published by DAAAM International, ISBN 9783-902734-11-2, ISSN 1726-9679, Vienna, Austria

DOI: $10.2507 / 28$ th.daaam.proceedings.074

\begin{abstract}
To provide increasing of effectiveness of industrial systems with achievement of the guaranteed product quality, the manufacturing digital transformation paradigm is applied. According to Industry 4.0 concept, adaptive, client-oriented automatic control of product life cycle is the basic approach in global manufacturing digitalization. Such technologies as Big Data, Cloud Services, advanced analytic algorithms, have to be implemented to provide an effective control for complex technological processes. Therefore, different technologies operating information from Web as well as from field level and top level manufacture information systems should offer high degree of interoperability.

This article represents the, so-called, digital twin concept description. The main development issues and paradigms are described, applying to oil refining industry.
\end{abstract}

Keywords: Industry 4.0; Digital Twin; Intelligent Control System; Automation; Global Digitalisation

\section{Introduction}

In the modern world, due to the high speed of IT technology growth, it appears more opportunities to improve the efficiency of traditional industrial systems and the quality of distributed products. Applying the paradigm of digital production, with the help of modern information technologies, it is possible to create digital twin of the whole manufactory, and specify its pieces as detached systems [1]. This is especially actual for industries distributed in space and time. The main problem is to build a digital twin for a separate control object, it is necessary to take into account the nature of the object and a number of basic properties such as:

- $\quad$ discreteness / continuity;

- linearity / nonlinearity;

- $\quad$ stationarity / nonstationarity;

- distribution / concentration of parameters;

- the presence of uncertainties in the description of the structure / parameters;

- $\quad$ stochasticity / determinism;

- multiple connectivity and so on. 
In this article, the following questions are considered:

- What does the term "Digital Twin" include?

- What parts do Digital Twins consist of?

- How to implement management based on a Digital Twin?

- How to build a Digital Twin using modern IT technologies such as Big Data, Cloud Services, and Advanced Analytic Algorithms?

\section{What is a Digital Twin}

The concept of digital twin envisages connection between the physical and digital world over the analytical program and machinery. The twin preimage of real model is generated at the design stage. Including the basic properties of all produced objects. The digital model and the physical object interact in real time [2]. This information exchange is characterized by a Big Data which generated by a multitude of sensors. The digital model is constantly updated. That is, it changes its parameters to better match the current working mode of the physical object. Thus, there is a real opportunity to identify emerging anomalies in the early stages, to predict the behaviour of the object and to ensure implementation algorithms of dynamic optimisation, which ultimately allows significantly improve the reliability and efficiency of the equipment [1, 3-5].

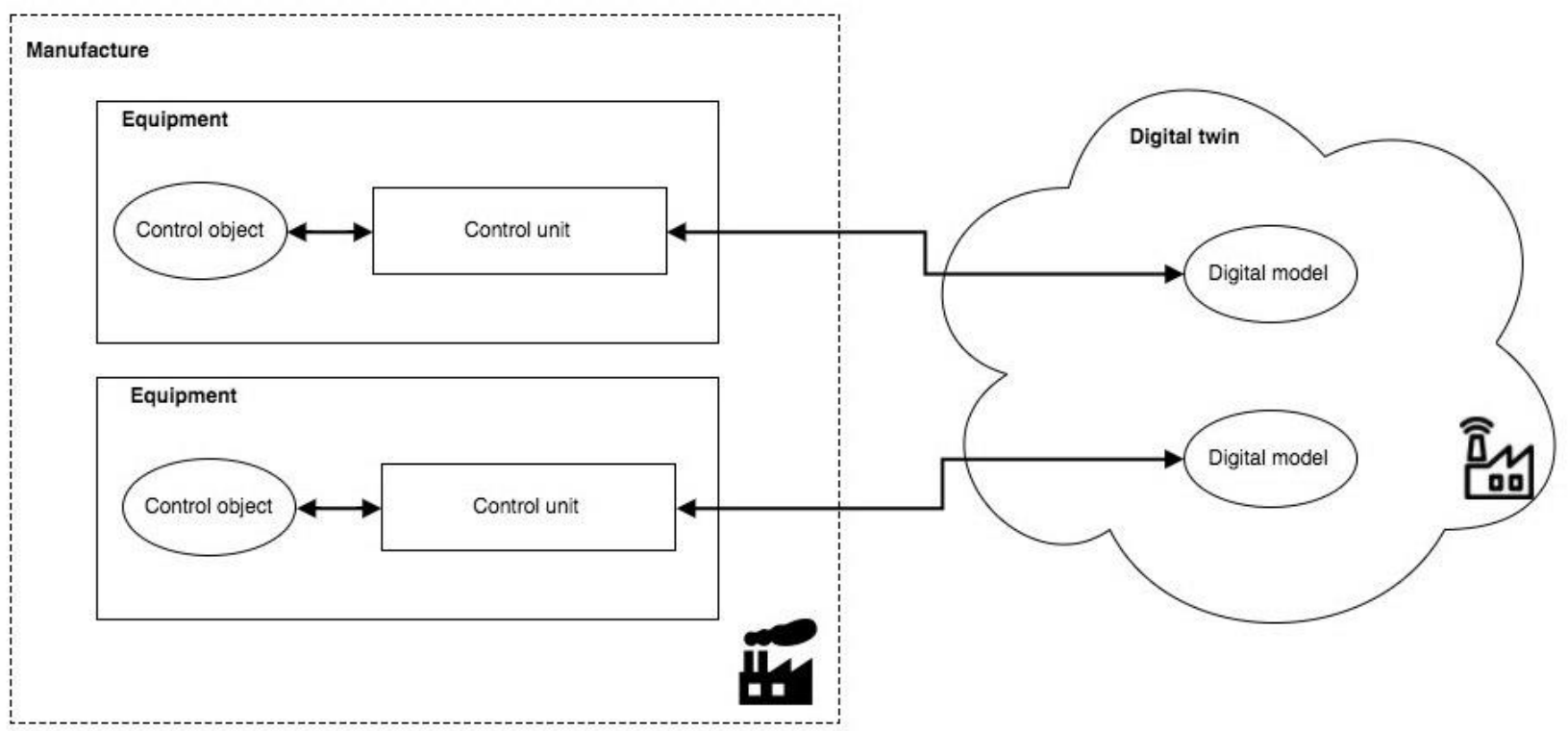

Fig. 1. Data exchange between a physical object and a digital model

\section{Digital Twin Units}

Digital twin - is the complex information and technical systems that cover practically all levels of the automated process control system (ACS): from the level of terminal equipment to the level of enterprise, resource planning (ERP). These levels are illustrated on Fig. 2.

In this article, we will consider digital twin as a multilayer system, consisting of five layers. Each of these levels represents itself as separate subsystem of Digital Twin. Development of each of them can be made independently, so this makes the process of development less complicated and provide multi-user access for developers [6]. Let us consider each level more precisely, Fig. 3.

\subsection{Cyber-physical layer}

At this level, the stabilization of the mode parameters and the program-logical control of the object are performed, which can be represented by separate installations or a technological process.

\subsection{Primary processing/store data layer}

This level, data are collected from devices represented by the cyber physical layer and their primary processing (for example: conversion of values) and temporary storage (for example: until the next layer is transferred). This layer can be represented by the implementation of OPC (Object Linking and Embedding for Process Control) [7]. 


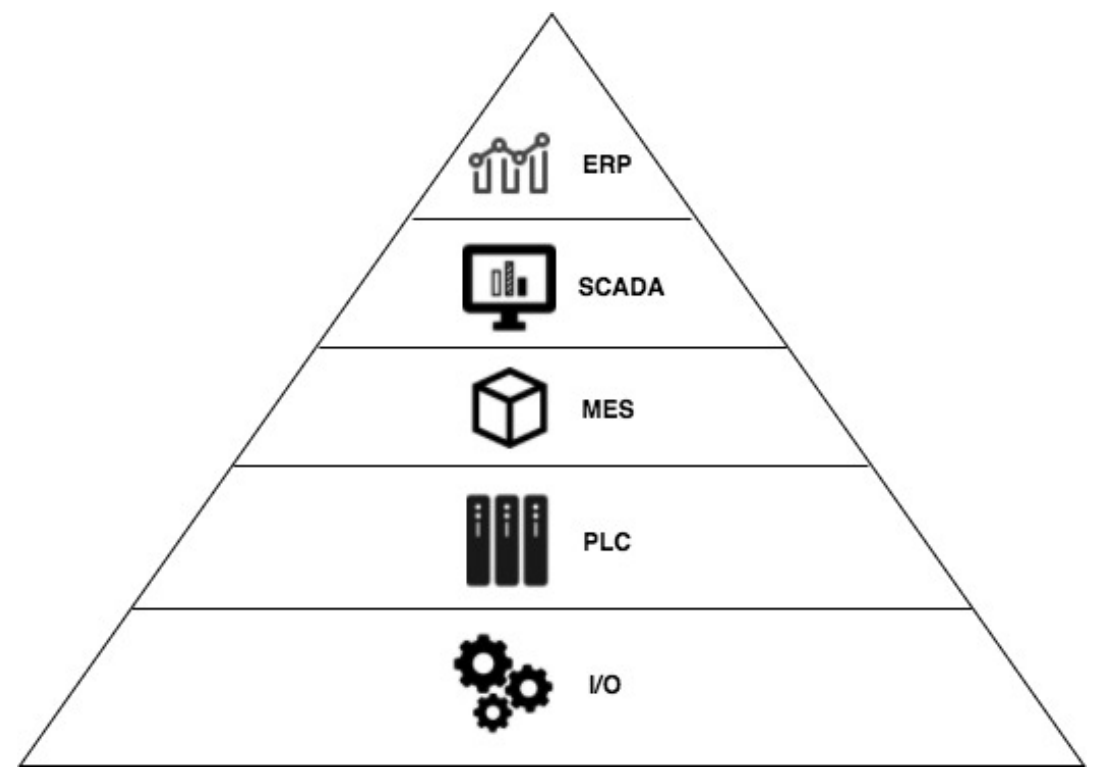

Fig. 2. Layers of the ACS

\subsection{Distributed computing and storage layer}

This layer is the kernel of the digital twin. It contains such units as:

- API (Application program interface). Through this interface we can communicate with multiple layers in the same time, using one of specified protocols (TCP, HTTP, WebSocket).

- Distributed computing management system parses tasks on huge number of subtasks and sends them to specified evaluation nodes [7]. Then it collects the results. So it is the way of representation of parallel evaluation approach.

- Distributed storage management system, provides the storage of technical data, master data, analysis results, etc. in on DDB. It can be represented by any DBMS(SQL, NoSQL, TSDB) for any type of data. (As an Example: for data from sensor - the Apache Cassandra can be used, but for project documentation- MongoDB, which is documentorientated) $[7,8]$

\subsection{Models and algorithms layer}

On this layer, mathematical, statistical, neural-network models and CAD models can be stored.

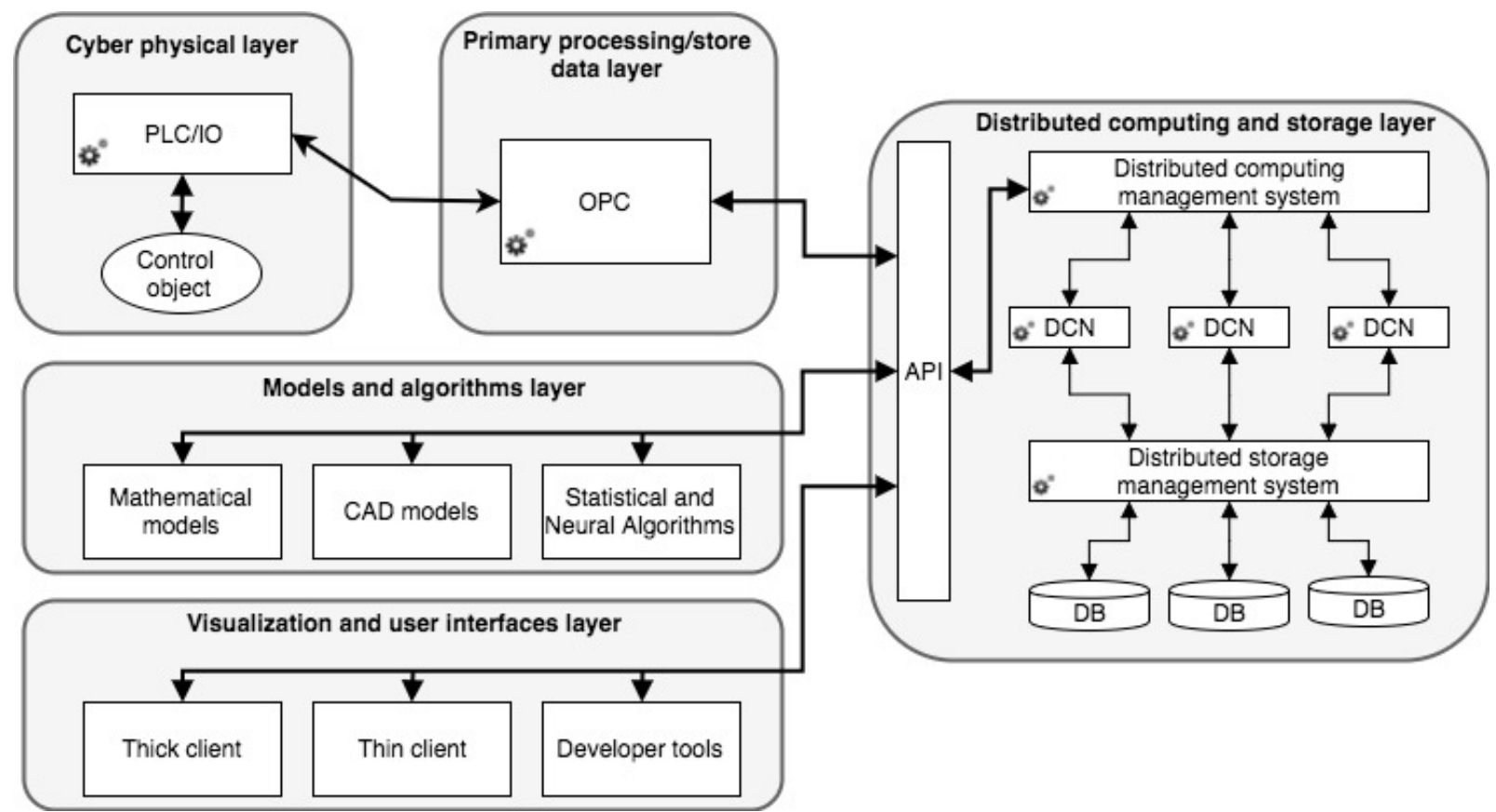

Fig. 3. Digital twin structure. 


\subsection{Visualisation and user interfaces layer}

This layer provides the access for users to digital twin, using graphical interface. It contains:

- $\quad$ Rich-client provides AWP interface as technical schemes, screens. Can be viewed as:

○ SCADA (Supervisory Control And Data Acquisition);

○ HMI (Human-machine interface);

- Thin-client (for example WEB - application) provides analytic screens interface for experts:

- Statistical model analysis

○ Object monitoring, according to dynamic of key parameter changes.

- Developer tools provides the tool for model and algorithm development. Can be represented with standalone specific application.

\section{Control with a digital twin}

A lot of digital twins functions are aimed at optimising control and making decision process:

- Identifying deviations in the current operation of equipment from the optimal mode;

- Identifying emergency situations;

- Alerting staff about events and situations;

- Analysing the causes of deviations

This functionality allows:

- Identify at an early stage of minor changes in the equipment, which in consequence can lead to serious damage and unscheduled downtime;

- Reduce the risk of failure of nodes and aggregates due to proactive diagnostics;

- Pre-alert operational staff of a potential threat to critical equipment failure;

- Reduce the time of unplanned downtime of equipment associated with its sudden breakage due to early warning and timely ordering of spare parts;

- Reduce the burden on operational personnel by attracting the attention of the operator only to statistically significant deviations in the parameters.

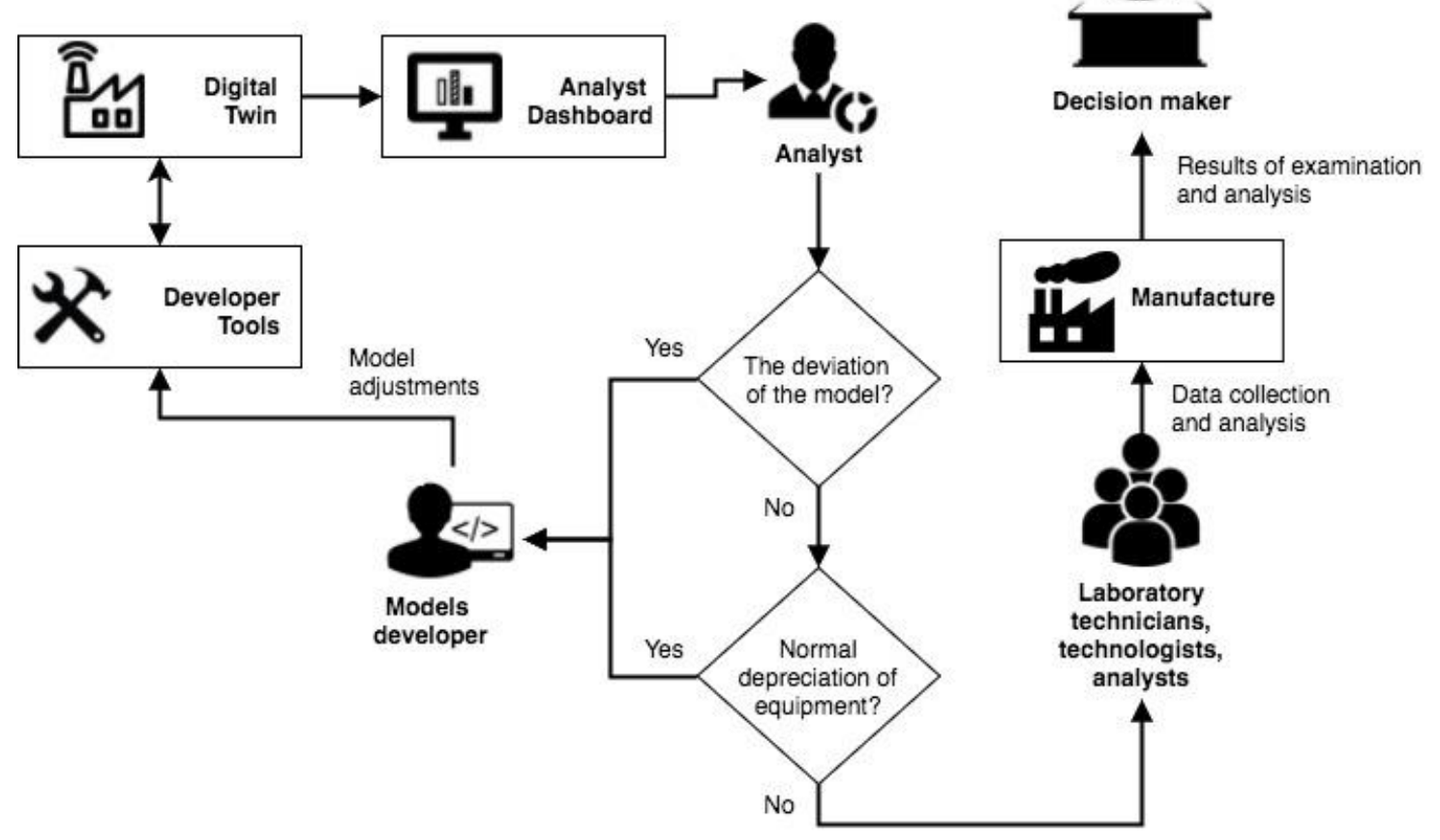

Fig. 4. Business decision-making process based on digital twin

The business decision-making process, when using the digital twin, can be represented, for example, in the same way as in Fig. 4. Thus, the process of equipment monitoring and diagnostics includes various production units from the operator and the instrumentation service to analysts and experts at the level of the company's main specialists [9]. 


\section{Building a Digital Twin}

As a basis for building a Digital Twin, we used the basic UML language and two profiles that define the objectoriented languages SysML and BPTL, proposed in the framework of OMG - Object Management Group. [10] SysML provides the ability to use 9 interrelated types of diagrams to describe the structure, behavior and system requirements:

- diagram of requirements;

- diagrams of activity (activities);

- diagrams of sequences;

- diagrams of states;

- diagrams of use cases (use cases);

- block definition diagrams;

- diagrams of internal block descriptions;

- diagrams of parameters;

- diagrams of packages.

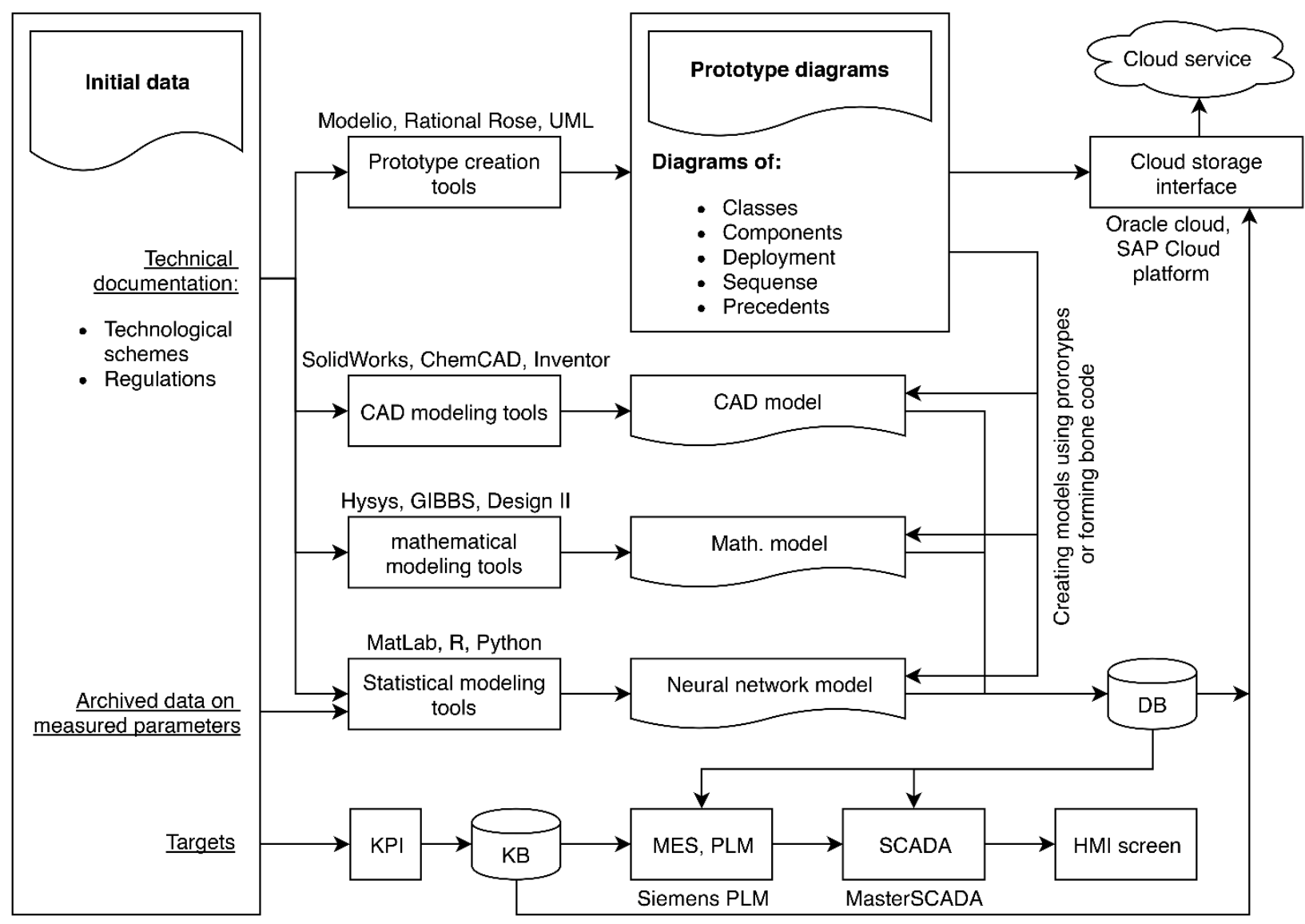

Fig. 5. The process of constructing the Digital Twin.

\section{Conclusion}

Digital Twin technology, which provides for the construction of "live" digital models and virtual simulators for local use or as an implementation of the Industrial Internet concept, is one of the key modern areas of operational analysis and increasing the efficiency of industrial equipment.

Expert and advisory diagnostic systems based on best practices can significantly reduce the costs of equipment maintenance, reduce the risks of their breakdowns, downtime and related material losses, and improve the efficiency and quality of technological processes. As a result of the development and verification of the architecture of Digital Twins, the problem of intelligent control system developing for distributed industrial object was solved. The results of the development of the architecture of digital twins are planned to be applied in one of the forming branches of Russia.

\section{Acknowledgments}

The article is published in the framework of the project Erasmus+ 573545-EPP-1-2016- 1-DE-EPPKA2-CBHE-JP and describes the part of the project conducted by SPbPU. 


\section{References}

[1] B. Katalinic, I. Kukushkin, V. Pryanichnikov, D. Haskovic, Cloud Communication Concept for Bionic Assembly System, Procedia Engineering, Volume 69, 2014, Pages 1562-1568, ISSN 1877-7058, doi:10.1016/j.proeng.2014.03.156

[2] Turner, C. J., Hutabarat, W., Oyekan, J. and Tiwari, A. (2016) 'Discrete Event Simulation and Virtual Reality Use in Industry: New Opportunities and Future Trends', IEEE Transactions on Human-Machine Systems, 46(6). doi: 10.1109/THMS.2016.2596099

[3] Scheifele, S., Friedrich, J., Lechler, A. and Verl, A. (2014) 'Flexible, Self-configuring Control System for a Modular Production System’, Procedia Technology. Elsevier B.V., 15, pp. 398-405. doi: 10.1016/j.protcy.2014.09.094.

[4] Kudriashov N., Protasov I., Markov S., Potekhin V., Yadgarova Y., Taratukhin V. (2016). Implementation of Cloud Services for Advance Management of Steel Transport for Continuous Casting Production, Proceedings of the 27th DAAAM International Symposium, pp.0457-0462, B. Katalinic (Ed.), Published by DAAAM International, ISBN 978-3-902734-08-2, ISSN 1726-9679, Vienna, Austria

[5] Gastermann, B., Stopper, M., Kossik, A., Katalinic, B. Secure Implementation of an On-Premises Cloud Storage Service for Small and Medium-sized Enterprises, Proceedings of DAAAM International Symposium on Intelligent Manufacturing and Automation, DAAAM 2014; Vienna; Austria; 26 November 2014 through 29 November 2014, Volume 100, Issue C, 2015, Pages 574-583

[6] Haskovic D., Katalinic B., Zec I., Kukushkin I., Zavrazhina A. (2016). Structure and Working Modes of the Intelligent Adviser Module, Proceedings of the 27th DAAAM International Symposium, pp.0866-0875, B. Katalinic (Ed.), Published by DAAAM International, ISBN 978- 3-902734-08-2, ISSN 1726-9679, Vienna, Austria

[7] Yerofeyev S.A., Ipatov O.A., Markov S.A., Potekhin V.V., Sulerova A.S. \& Shkodyrev V.P. (2015) Adaptive Intelligent Manufacturing Control System, Proceedings; DAAAM International Symposium of Intelligent Manufacturing and Automation, DAAAM 2015; Vienna; Austria; Volume 26, No. 1, ISSN 2304-1382

[8] Haskovic, D., Katalinic, B., Kukushkin, I. Role o. The Adviser Module i. The hybrid assembly subordinating control structure, Proceedings of DAAAM International Symposium on Intelligent Manufacturing and Automation, DAAAM 2014; Vienna; Austria; 26 November 2014 through 29 November 2014, Volume 100, Issue C, 2015, Pages 1706-1713

[9] Yerofeyev S., Ipatov O., Markov S., Potekhin V., Sulerova A., Shkodyrev V. (2016). Adaptive Intelligent Manufacturing Control Systems, Proceedings of the 26th DAAAM International Symposium, pp.1016-1024, B. Katalinic (Ed.), Published by DAAAM International, ISBN 978-3-902734-07-5, ISSN 1726-9679, Vienna, Austria

[10] Rosen, R., Von Wichert, G., Lo, G. and Bettenhausen, K. D. (2015) 'About the importance of autonomy and digital twins for the future of manufacturing', IFACPapersOnLine. Elsevier Ltd., 28(3), pp. 567-572. doi: 10.1016/j.ifacol.2015.06.141. 